\title{
The use of a combined profunda femoris perforator-based fasciocutaneous flap and gracilis muscle flap in the treatment of ischial pressure wounds in patients with limited mobility
}

\author{
Siobhan Fitzpatrick ${ }^{1,2}$ (1) $\cdot$ Chris Ahn ${ }^{1} \cdot$ Jeon Cha ${ }^{1} \cdot$ Megan Hassall ${ }^{1} \cdot$ Rowan Gillies $^{1} \cdot$ Jack Zoumaras $^{1} \cdot$ \\ John Vandervord ${ }^{1}$
}

Received: 12 January 2019 / Accepted: 27 February 2019 / Published online: 19 March 2019

(C) The Author(s) 2019

\begin{abstract}
Background Although general principles of pressure wound management begin with optimisation of patient and wound factors to promote healing, refractory cases may require surgical intervention. Compared with other areas, ischial tuberosity (IT) wounds tend to occur in wheelchair-bound patients and may respond well to surgical debridement and flap reconstruction where added tissue bulk is preventative for further pressure ulcer formation. IT ulcers may occur in patients with some degree of ambulatory potential or those who have a temporary impairment. While the most commonly used flap for typical IT ulcers is the musculocutaneous hamstring flap, for these patients, this is not suitable due to the sacrifice of muscle function. We designed a combined posterior thigh flap with pedicled gracilis to provide robust coverage and vascularised muscle bulk, while preserving hamstring function and potential for re-advancement in ulcer recurrence.

Methods Patients were selected for their grade of ulcer, compliance with physiotherapy, and their need to preserve muscle function. Each underwent surgical debridement and reconstruction using the combination flap. End-points included wound coverage, post-operative mobility, and complications. Follow-up was 3 months post-surgery.

Results All patients achieved complete coverage of their wound. In the follow-up period, there were no wound complications or ulcer recurrence.

Conclusion We describe a novel reconstruction method for IT pressure ulcers that maintains patient mobility. This combination perforator-based fasciocutaneous and gracilis flap is a superior reconstructive option that has reduced donor site morbidity and relatively simple operative technique and can be reutilised in ulcer recurrence.

Level of Evidence: Level V, therapeutic study.
\end{abstract}

Keywords Ischial pressure ulcer · Perforator flap · Gracilis flap

\section{Introduction}

Pressure wounds present an important challenge to patient care, being a source of numerous complications and often resulting in prolonged hospital admissions and need for a long-term follow-up. Patients at risk for development of pressure ulcers include those with multiple comorbidities such as

\section{Siobhan Fitzpatrick}

$1 \quad$ Plastic and Reconstructive Surgery Department, Royal North Shore Hospital, Reserve Road, St Leonards, NSW 2065, Australia

2 West Pennant Hills, Australia diabetes, dementia, stroke, peripheral vascular disease, and urinary incontinence - combined with extrinsic factors such as impaired mobility, whether temporary or permanent $[1,2]$.

After optimisation of causative factors, the surgical management of Grade III and IV pressure ulcers usually involves wide surgical debridement and, in the appropriate patient, subsequent flap repair [1, 3, 4]. Flap selection should include adequate soft tissue coverage to fill the defect, providing healthy and vascularised tissue to the wound base, closing off dead space for potential collections, while maintaining a potential for further re-advancement considering the high rates of ulcer recurrence [5-7].

Amongst pressure ulcer sites, ischial tuberosity (IT) ulcers usually occur due to prolonged seated positioning and can therefore occur in otherwise healthy patients 
who fail to successfully offload pressure points. These are some of the best candidates for flap reconstruction, and the hamstring advancement technique as described by Hurteau et al. has become the mainstay for IT pressure sore reconstruction [3].

There is however a small subset of IT pressure ulcer patients who have some degree of residual mobility or potential for regaining ambulation and return to walking status. In this group, a hamstring sacrificing reconstruction is not suitable as the hip extensors provide a critical role in normal gait. A secondary option, the fasciocutaneous posterior thigh flap alone, often fails to provide adequate tissue bulk to fill the defect and therefore allows for skin closure only without addressing underlying dead space and therefore inadequate control of residual infection.

In relative proximity to the wound location, the gracilis muscle is a readily available and expendable muscle that is well described in its use as a pedicled or free option for reconstruction $[8,9]$. The dissection is relatively straightforward, provides adequate bulk of well-vascularised tissue, and does not affect mobility.

We present three cases of reconstruction using a profunda femoris perforator flap based on the posterior thigh fasciocutaneous design, combined with a pedicled gracilis transposition in patients with potential return to mobility and their long-term outcomes.

\section{Methods}

Three patients were selected for this novel surgical reconstruction. Each patient had developed a pressure ulcer after a temporary, but prolonged period of limited mobility. There was a need to preserve hip extensors for mobility, while the size and depth of their ischial pressure wound required more than fasciocutaneous coverage alone. In selecting these patients, the level of compliance required immediately after surgery and with subsequent allied health therapy was taken into consideration. Criteria included the compliance to remain off the area for 2 weeks while maintaining preventative pressure offloading to other areas. Patients received education regarding pressure care and restrictions that would be required postoperatively.

The post-operative protocol was a 2-week period of strict prone or lateral decubitus positioning. Drains were monitored and removed at 2 weeks following an ultrasound to confirm the absence of underlying collections. Patients were started on a graduated seating protocol with allied health over the following 2 weeks after which they could remain seated on the reconstructed area for up to $10 \mathrm{~h}$. All patients were followed up for a period of 3 months post-operatively, and there were no incidences of wound-related complications or recurrence of pressure areas.

\section{Relevant anatomy}

After branching from the femoral artery within the upper thigh, the profunda femoris artery gives off a medial and lateral circumflex femoral branch and continues on to give off four perforating arteries which supply the deep structures of the thigh, including the posterior compartment and overlying skin.

In particular, the first and second perforating arteries have cutaneous branches that travel to the posteromedial aspect of the thigh. The design of the posterior thigh flap skin paddle is located over these perforators (Fig. 1), and while the dominant supply is from the inferior gluteal artery from above, the flap can also be raised on these secondary perforators with good vascularity.

Supplied by the aforementioned medial circumflex femoral artery as its dominant pedicle, the gracilis muscle arises from the pubic ramus and inserts as one of three muscles in the pes anserinus into the medial tibial condyle. The muscle is a type II muscle, allowing adequate perfusion via its dominant vessel even after division of its more distal minor arterial supply (Fig. 2).

\section{Operative technique}

Pre-operatively, a large V-Y or medially based hatchet design skin paddle is marked spanning the length of the posterior thigh and at least as wide as the defect. The midline of the thigh is the axis of the flap, and a hand-held doppler is used to

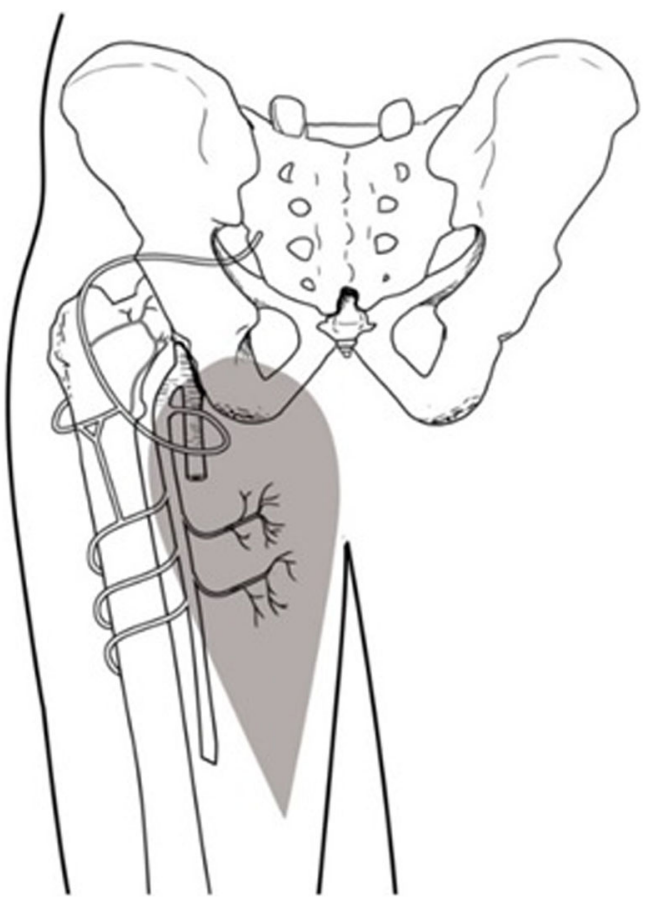

Fig. 1 Diagram of the planned fasciocutaneous posterior thigh flap based on the upper two perforators from the profunda femoris artery 


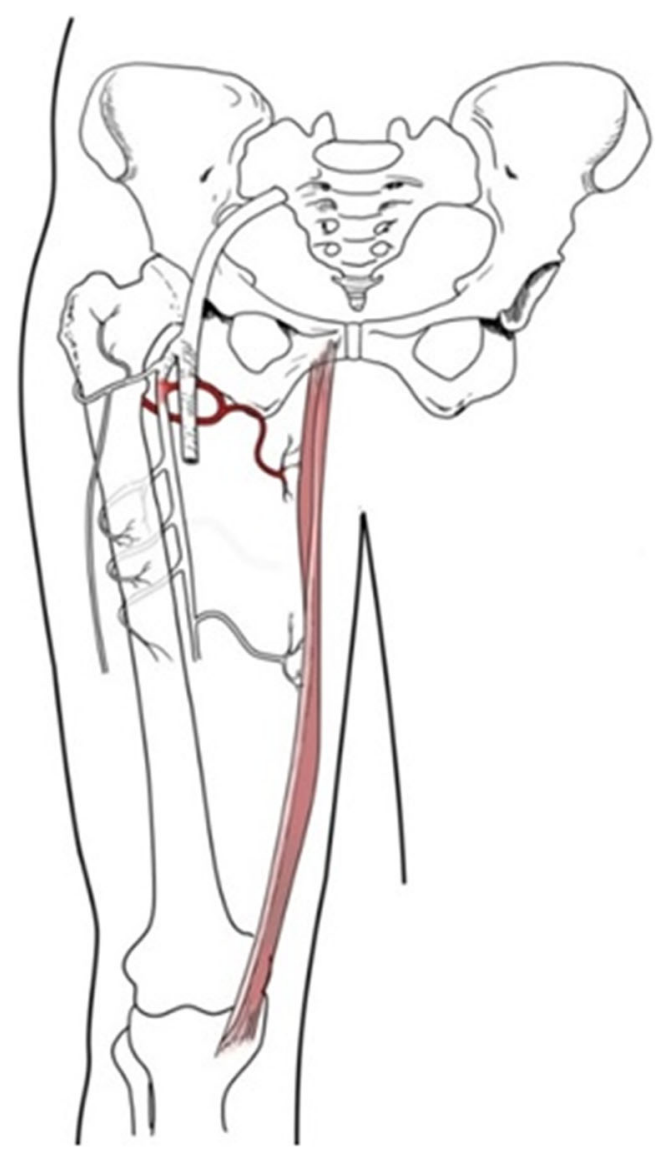

Fig. 2 Diagram illustrating gracilis muscle and its pedicle branching from the median circumflex femoral artery

identify perforators from the profunda femoris artery along this line. The location of gracilis is also marked in a line from the pubic tubercle to the medial tibial condyle approximately $2 \mathrm{~cm}$ posterior to the line of the adductor sulcus in the inner thigh. This can be difficult in the overweight patient and the surgeon should rely on careful dissection especially in the prone position to identify and confirm gracilis prior to distal division and further mobilisation.

The procedure is performed with the patient positioned prone (Fig. 3), commencing with pseudo-tumour excision of the pressure ulcer. The marked incisions are then made to the sub-fascial plane and the fasciocutaneous flap component mobilised while taking care to preserve the upper two profunda perforators. If mobility is inadequate, the perforators should be dissected further through the posterior compartment of the thigh to allow further advancement.

The chimeric gracilis part of the flap is then dissected in continuity medially in the same sub-fascial plane. Once identified, the distal gracilis insertion is divided (Fig. 4). The dominant pedicle is confirmed entering the muscle at $10 \mathrm{~cm}$ inferior to the pubic tubercle in the middle third prior to division of the distal vasculature. The dissection should not require division of the proximal origin as adequate length of muscle should reach an ischial defect; however, if short, this option

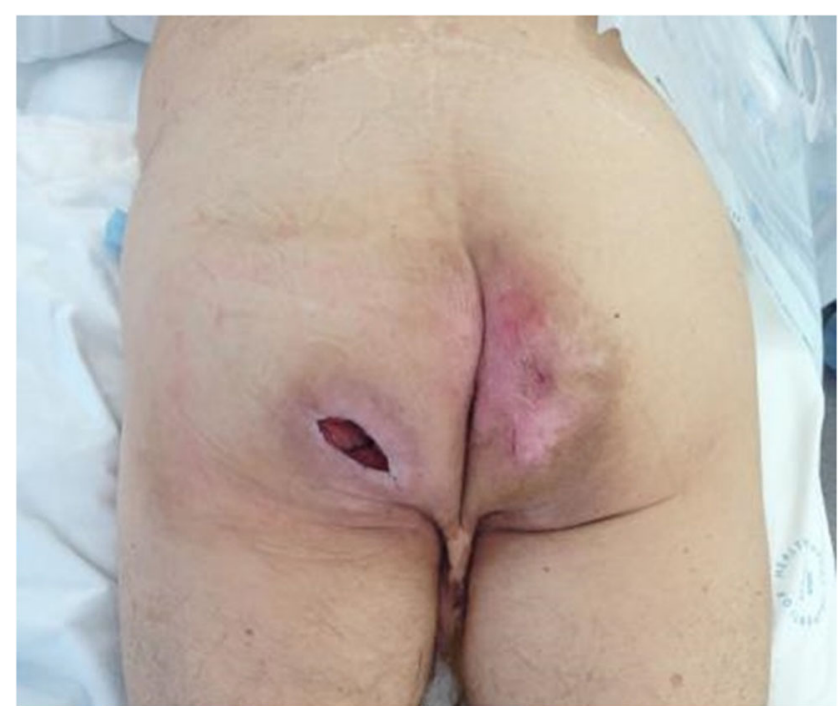

Fig. 3 Case 3 patient positioned prone with Grade IV pressure ulcer visible

would allow further mobility. The muscle is then transposed into the defect and loosely inset to cover exposed bone and fill in dead space (Figs. 5 and 6). The posterior thigh flap can now be advanced over this to provide skin closure.

$3 \times 15$ French suction drains are used with one drain in the ischial defect region while the remaining two are placed either side of the posterior thigh flap (Fig. 7). Closure is performed in layers with simple dressings changed daily over the wounds.

\section{Results}

\section{Case 1}

Thirty-two-year-old male with Spina Bifida (L2-5 myelomeningocele) presented with a recurrent right ischial tuberosity pressure wound. Initial debridement and application of

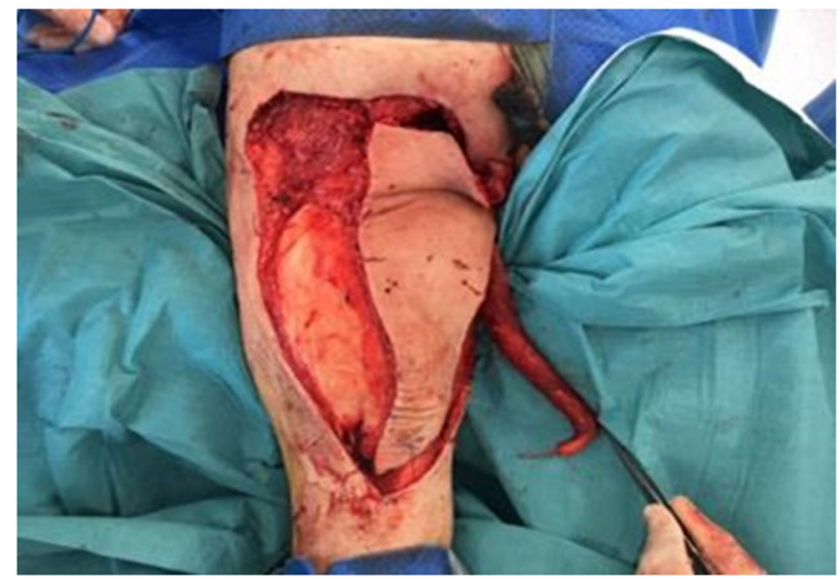

Fig. 4 Case 3 fasciocutaneous posterior thigh flap dissected down to subfascial plane and the gracilis muscle drawn out medially by its detached distal insertion 


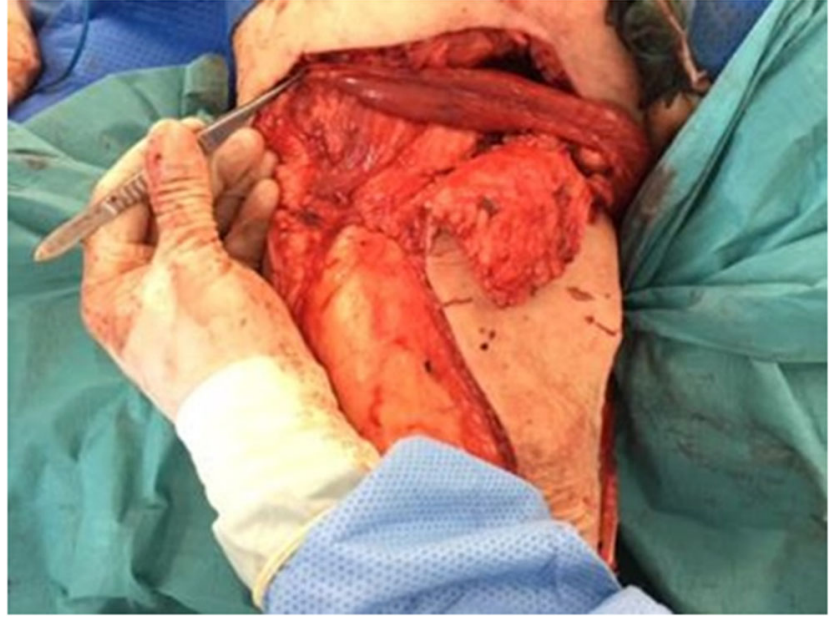

Fig. 5 Case 3 gracilis muscle is folded laterally into the defect to provide muscular bulk and eliminate dead space

VAC dressing were performed in theatre to assess suitability of the wound. After 2 weeks of wound care and optimisation, the patient underwent a pseudo-tumour excision of the right ischial tuberosity pressure ulcer, with immediate combined posterior thigh and gracilis flap reconstruction. Post-operative antibiotic prophylaxis was administered until discharge. Routine ultrasound of the right thigh at 2 weeks showed no collection, and drains were removed the following day. Patient commenced a staged seating protocol from post-operative day 17 , and he was progressively seated and mobilised by physiotherapy until suitable for discharge to rehabilitation facility at day 25 . His mobility status was equivalent to pre-procedure levels as assessed by physiotherapy and occupational therapy.

\section{Case 2}

Forty-five-year-old female with T12 paraplegia presented with a chronic right ischial tuberosity pressure wound.

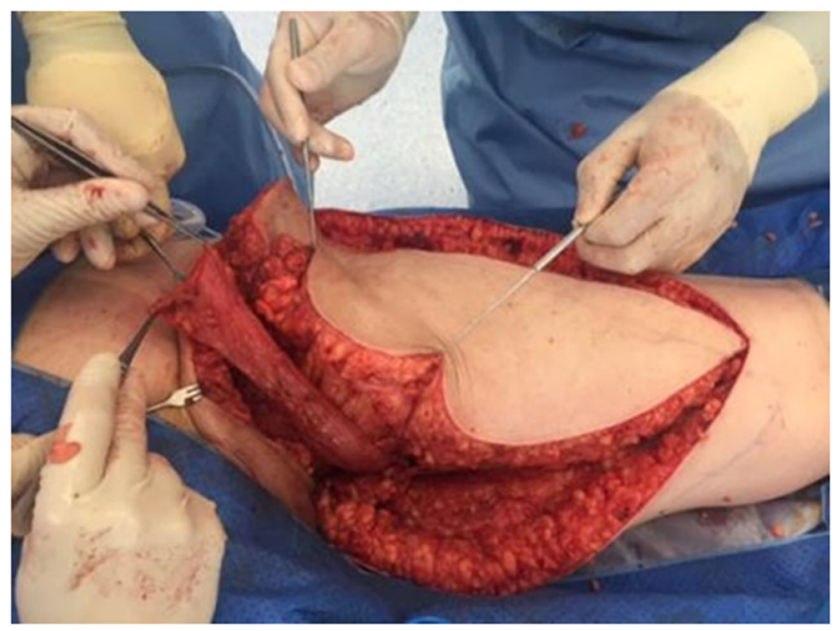

Fig. 6 Case 1 fasciocutaneous posterior thigh flap has been dissected out, and pedicled gracilis is folded laterally into the defect

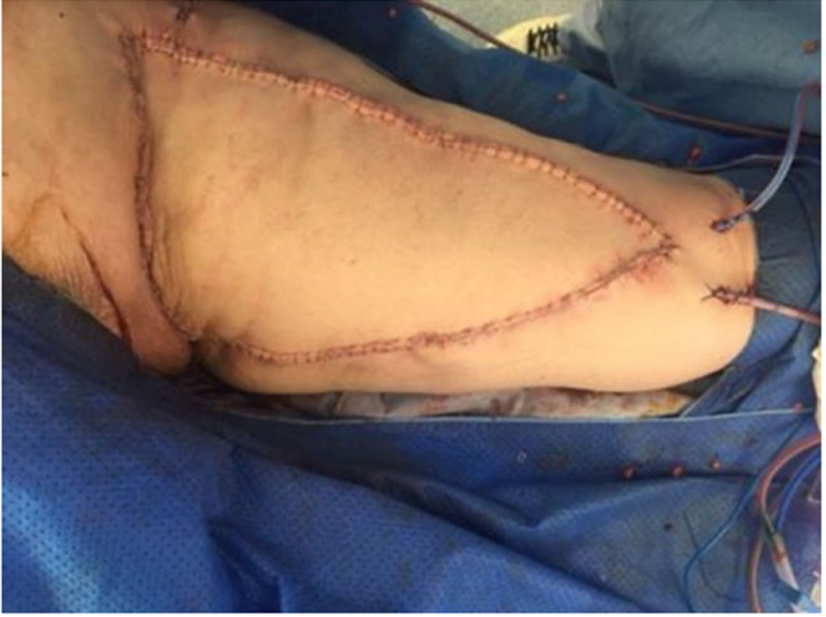

Fig. 7 Case 1 after skin closure with surgical drains placed appropriately

Patient underwent a pseudo-tumour excision and debridement of ischial tuberosity pressure sore followed by a combined pedicled gracilis flap and V-Y posterior thigh flap. Patient was treated with vancomycin IV for 4 weeks. Her routine right thigh ultrasound at 2 weeks showed a small collection, and a drain was inserted under ultrasound guidance, draining $40 \mathrm{ml}$. Drains remained in situ for a further 2 weeks. Repeat ultrasound showed minimal collection, and drains were removed and seating protocol commenced. Although this patient's mobility was limited prior to the operation, she wished to preserve any potential to regain ambulatory gait after the procedure which was accounted for in operative planning.

\section{Case 3}

Sixty-five-year-old male with Spina Bifida presented with a recurrent left ischial tuberosity pressure wound. Patient underwent a pseudo-tumour excision of the ischial tuberosity wound plus V-Y posterior thigh flap repair with pedicled gracilis flap. At 2 weeks post-operation, ultrasound showed no collections and drains were removed. Patient commenced seating protocol at 14 days with physiotherapy. His postoperative mobility with four-wheeled walker was returned to near baseline, and the patient was cleared by physiotherapy for discharge.

In all three of these cases, the use of a combined fasciocutaneous posterior thigh flap and pedicled gracilis flap was a successful reconstructive option. Outcomes were assessed in the peri-operative phase and at 3 months. For a summary of outcomes, see Table 1 .

\section{Discussion}

Pressure wounds can occur in any patients with limited mobility, whether temporary or permanently impaired. Ischial 
Table 1 Surgical and functional outcomes for three patients undergoing reconstruction with a combined fasciocutaneous posterior thigh flap and pedicled gracilis flap

\begin{tabular}{ll}
\hline Outcomes & Occurrence \\
\hline Inadequate coverage & Nil \\
Flap collection & 1 (resolved with drain) \\
Wound breakdown & Nil \\
Infection & Nil \\
Ulcer recurrence & Nil \\
Morbidity & Nil \\
Mortality & Nil \\
Decline in mobility & Nil \\
\hline
\end{tabular}

pressure sores account for $28 \%$ of all such ulcers [10]. The management of Grade III and IV pressure ulcers involves optimisation of causative factors, local wound care, and for refractory cases, surgical intervention with debridement and in suitable patients, flap reconstruction.

In ambulatory patients, the choice of flap not only needs to provide adequate soft tissue coverage and the potential for readvancement, but it should also preserve pre-operative mobility. The combination of a fasciocutaneous posterior thigh flap and pedicled gracilis flap was a successful reconstructive option in these three cases involving patients with limited mobility.

The most commonly used flap for the treatment of ischial tuberosity pressure sores is a musculocutaneous hamstring flap as described by Hurteau et al. in 1981. This workhorse flap has the advantage of providing large volume tissue bulk and well-vascularised muscle, with the robustness of a skin paddle that provides cushioning and stability against shear and moisture. Multiple variations of the hamstring advancement flap have been proposed throughout the years yet all rely on the dissection and sacrifice of the hamstring muscles and their consequential loss of function [3, 11-14].

As surgical techniques improve and the need arises for more versatile flaps associated with low morbidity, the concept of perforator flaps was introduced. Initially described by Kroll et al. in the late 1980 s, perforator flaps such as the profunda artery-based fasciocutaneous flap are being increasingly used, and some authors report a lower rate of recurrence [15-18].

The success of this profunda artery-based fasciocutaneous flap may be attributed to its reliable perfusion, its increased versatility compared to muscular pedicles, and its ability to be re-advanced [18, 19]. Most importantly, this flap also retains the function of the underlying musculature, overcoming the principle disadvantage of the V-Y hamstring advancement flap, and preserving patient pre-surgical mobility [4, 19, 20].

The main disadvantage of this flap, however, is in its limited ability to obliterate dead space. In those patients with large defects, the posterior thigh flap alone cannot provide adequate bulk to reconstruct the missing tissue [4]. Ultimately when used alone, these flaps only provide soft tissue cover for minor to moderately sized ischial pressure ulcers [21].

Gracilis muscle flaps have been used extensively throughout the years in a variety of free and pedicled flap applications [8]. The gracilis is an easily attained muscle flap with a reliable blood supply and abundant flow, making it a robust reconstructive flap that may help to control residual infection and promote healing in ischial tuberosity wounds [9]. The mass of tissue provided by the gracilis flap effectively eliminates dead space and may allow for better distribution of pressure on return to seating [22]. As the gracilis is not essential for normal gait, it serves as a viable reconstructive option in patients with limited mobility.

By enhancing the profunda-based fasciocutaneous flap with an additional pedicled gracilis flap, we aimed to combine the advantages of each flap type while overcoming the limitations of the traditional reconstructive approach. Using this technique, we were able to design a versatile flap with wellperfused skin closure, while having a separate robustly perfused muscle component to fill the dead space postdebridement and control infection, all while preserving ambulatory function. Our operative technique also improves on that described by Lee and colleagues, as we complete the entire operation with the patient in the prone position rather than supine for gracilis harvest and prone for flap placement and closure [4].

Mobility is considered to be the most important contributor to quality of life in patients with Spina Bifida [23]. Of the three patients examined in this series, each individual expressed a strong desire to maintain their mobility, or in the second case, the potential to improve mobility status through rehabilitation. In our increasingly ageing population and with greater numbers of those being placed in care facilities, the incidence of pressure wounds in patients with limited mobility is on the rise [1]. This means that the need for mobility preserving options of flap repair will be increasingly relied upon, and the combination flap in this series may be considered as a suitable reconstructive option.

The principle underlying this combination flap also lends itself to the development of various other reconstructive options. The transposed fasciocutaneous flap and a rotated split-muscle gluteus maximus flap, as described by Borgognone and colleagues, illustrate the use of a partial thickness muscle flap which precludes functional loss [24]. This flap has the advantage of preserving most of the gluteus maximus muscle for stair climbing and single-limb support in the ambulatory patient, while still providing adequate bulk for pressure wound defects. This and other reconstructive flap options in ambulatory patients should be further developed to ensure that the plastic and reconstructive surgeon is well equipped to deal 
with these complex pressure wounds that cause a significant burden on our health system.

Unfortunately, patients with ischial tuberosity wounds are at high risk of ulcer recurrence, with some studies reporting recurrence rates of up to $48 \%$, so it is important to provide patient education for pressure avoidance, skin care, and cushioning [7]. Those flap designs, as in our combined flap, should maintain the potential for re-advancement if a recurrence should occur.

\section{Conclusion}

Ischial pressure ulcers are the most common wounds in patients with limited mobility. Here, we describe a novel method in which to cover Grade III and IV pressure ulcers while maintaining mobility using a combined profunda artery perforator flap and pedicled gracilis flap. We believe that this is a suitable reconstructive option with benefits of introducing wellvascularised muscle tissue with robust skin coverage while minimising donor morbidity with a relatively simple operative technique that can be reutilised in cases of recurrence.

Funding information No funding was received for this article.

\section{Compliance with ethical standards}

Conflicts of interest Dr. Siobhan Fitzpatrick, Dr. Chris Ahn, Dr. Jeon Cha, Dr. Megan Hassall, Dr. Rowan Gillies, Dr. Jack Zoumaras, and Dr. John Vandervord have no conflicts of interest.

\section{Ethical approval Not required.}

Patient consent Informed consent was obtained from all individual participants included in the study.

Open Access This article is distributed under the terms of the Creative Commons Attribution 4.0 International License (http:// creativecommons.org/licenses/by/4.0/), which permits unrestricted use, distribution, and reproduction in any medium, provided you give appropriate credit to the original author(s) and the source, provide a link to the Creative Commons license, and indicate if changes were made.

\section{References}

1. Gusenoff J, Redett R, Nahabedian M (2002) Outcomes for surgical coverage of pressure sores in nonambulatory, nonparaplegic, elderly patients. Ann Plast Surg 48:633-640

2. Allman R (1997) Pressure ulcer prevalence, incidence, risk factors, and impact. Clin Geriatr Med 13:421-436

3. Hurteau J, Bostwick J, Nahai F, Hester R, Jurkiewicz M (1981) V-Y advancement of hamstring musculocutaneous flap for coverage of ischial pressure sores. Plast Reconstr Surg 68:539-542

4. Lee S, Huang S, Chen M, Chang K, Lai C, Lin S (2009) Management of recurrent ischial pressure sore with gracilis muscle flap and V-Y profunda femoris artery perforator-based flap. J Plast Reconstruct Aesthet Surg 62:1339-1346

5. Foster R, Anthony J, Mathes S, Hoffman W (1997) Ischial pressure sore coverage: a rationale for flap selection. Br J Plast Surg 50:374-379

6. Kroll S, Hamilton S (1988) Multiple and repetitive uses of the extended hamstring V-Y myocutaneous flap. Plast Reconstr Surg 84:296-302

7. Tavakoli K, Rutkowski S, Cope C, Hassall M, Barnett R, Richards M, Vandervord J (1999) Recurrence rates of ischial sores in paraand tetraplegics treated with hamstring flaps: an 8-year study. Br J Plast Surg 52:476-479

8. Labandter H (1980) The gracilis muscle flap and musculocutaneous flap in the repair of perineal and ischial defects. Br J Plast Surg 22:95-98

9. Wingate G, Friedland J (1978) Repair of ischial pressure ulcers with gracilis myocutaneous island flaps. Plast Reconstr Surg 62:245-248

10. Dansereau J, Conway H (1964) Closure of decubiti in paraplegics: report on 2000 cases. Plast Reconstr Surg 33:474-480

11. McCraw J, Dibbell D, Carraway J (1977) Clinical definition of independent myocutaneous vascular territories. Plast Reconstr Surg 60:341-352

12. Tobin G, Sanders B, Man D, Weiner L (1981) The biceps femoris myocutaneous advancement flap: a useful modification for ischial pressure ulcer reconstruction. Ann Plast Surg 6:396-401

13. Quaba A, Chapman R, Hackett M (1986) Extended application of the biceps femoris musculocutaneous flap. Plast Reconstr Surg 81: 94-105

14. Cope C, Barry P, Hassall M, Barnett R, Richards M, Vandervord J (1995) V-Y advancement hamstring myocutaneous island flap repair of ischial pressure ulcers. Aust N Z J Surg 65:412-416

15. Kroll S, Rosenfield L (1988) Perforator-based flaps for low posterior midline defects. Plast Reconstr Surg 81:561-566

16. Koshima I, Moriguchi T, Soeda S, Kawata S, Ohta S, Ikeda A (1993) The gluteal artery perforator-based flap for repair of sacral pressure sores. Plast Reconstr Surg 91:678-683

17. Homma K, Murakami G, Fujioka H, Fujita T, Imai A, Ezoe K (2001) Treatment of ischial pressure ulcers with a posteriomedial thigh fasciocutaneous flap. Plast Reconstr Surg 107:1725-1731

18. Lin C, Ma H (2012) Perforator-based fasciocutaneous flap for pressure sore reconstruction. J Plast Surg Hand Surg 46:430-433

19. Rubin J, Whetzel T, Stevenson T (1995) The posterior thigh fasciocutaneous flap: vascular anatomy and clinical application. Plast Reconstr Surg 95:1228-1239

20. Lin C, Ma H (2016) Use of perforator-based fasciocutaneous flaps for pressure sore reconstruction: single-perforator-based versus multiple-perforator-based flaps. Aesthet Plast Surg 40:540-548

21. Ichioka S, Okabe K, Tsuji S, Ohura N, Nakatsuka T (2004) Triple coverage of ischial ulcers with adipofascial turnover and fasciocutaneous flaps. Plast Reconstr Surg 114:901-905

22. Kim C, Yun I, Lee D, Rah D, Lee W (2014) Treatment of ischial pressure sores with both profunda femoris artery perforator flaps and muscle flaps. Arch Plast Surg 41:387-393

23. Schoenmakers M, Uiterwaal C, Gulmans V, Gooskens R, Helders P (2005) Determinants of functional independence and quality of life in children with spina bifida. Clin Rehabil 19:677-685

24. Borgognone A, Anniboletti T, De Vita F, Schirosi M, Palombo P (2010) Ischiatic pressure sores: our experience in coupling a splitmuscle flap and a fasciocutaneous flap in a 'criss-cross' way. Spinal Cord 48:770-773

Publisher's note Springer Nature remains neutral with regard to jurisdictional claims in published maps and institutional affiliations. 